\title{
Inclusive Searches for Squarks and Gluinos with the ATLAS Detector
}

\section{Moritz Backes*}

On behalf of the ATLAS Collaboration

University of Geneva, Switzerland

E-mail: Moritz.Backes@cern.ch

Despite the absence of experimental evidence, weak scale supersymmetry remains one of the best motivated and studied Standard Model extensions. This report summarises recent results on inclusive searches for supersymmetric squarks and gluinos in events containing jets, missing transverse momentum with or without leptons. The searches use the full data sample recorded in 2011 at $\sqrt{s}=7 \mathrm{TeV}$ centre-of-mass energy by the ATLAS experiment at the LHC.

36th International Conference on High Energy Physics,

July 4-11, 2012

Melbourne, Australia

${ }^{*}$ Speaker. 


\section{Introduction}

Supersymmetry (SUSY) is a candidate theory for physics beyond the Standard Model (SM). If R-parity conserving SUSY exists and it is broken at energies accessible by the Large Hadron Collider (LHC), squarks and gluinos are expected to be produced in pairs with relatively large cross-sections compared to other SUSY production processes. In the minimal supersymmetric extension of the SM (MSSM) such particles then decay into jets, leptons, and the lightest supersymmetric particle (LSP). Jets arise in the decays of squarks and gluinos, while leptons can arise in decays involving charginos or neutralinos. A stable, weakly interacting LSP will escape detection, leading to missing transverse momentum ( $p_{\mathrm{T}}^{\text {miss }}$ and its magnitude $E_{\mathrm{T}}^{\text {miss }}$ ) in the final state.

In the following, three inclusive searches for squarks of the first two generations and gluinos are presented: Two fully hadronic analyses with $E_{\mathrm{T}}^{\text {miss }}$ and jets with different multiplicities in the final state as well as one analysis requiring in addition one isolated lepton. The searches are based on $4.7 \mathrm{fb}^{-1}$ of data collected with the ATLAS detector [1] in 2011 at $7 \mathrm{TeV}$ center of mass energy in proton-proton collisions. The results are interpreted in the $\tan \beta=10, A_{0}=0, \mu>0$ slice of CMSSM/MSUGRA [2] parameter space and in various simplified models. Particular effort has been made to increase the sensitivity to models with compressed mass spectra, which suffer from small signal acceptances due to the low transverse momenta of their decay products.

\section{Searches in final states with 2-6 jets and missing transverse energy}

This search for SUSY particles decaying to fully hadronic final states [3] is based on triggers requiring jets and $E_{\mathrm{T}}^{\mathrm{miss}}$ in coincidence. Events with electrons or muons are vetoed. A number of inclusive channels with increasing jet multiplicity is defined to obtain the best discovery reach over the broad range of gluino and squark masses. The final discriminating variable is the inclusive effective mass $\left(M_{\text {eff }}^{\text {inc }}\right)$ defined as the scalar sum of the $E_{\mathrm{T}}^{\text {miss }}$ and the transverse momenta $\left(p_{\mathrm{T}}\right)$ of all jets with $p_{\mathrm{T}}>40 \mathrm{GeV}$ in the event. Each jet multiplicity channel has up to 3 signal regions (SR) which are defined by loose, medium, or tight selections on $M_{\mathrm{eff}}^{\mathrm{inc}}$. In addition, a dedicated SR for compressed SUSY scenarios is defined. This SR relies on the presence of an initial state radiation jet, against which the system of pair produced SUSY particle recoils. The resulting boost enhances the $E_{\mathrm{T}}^{\text {miss }}$ signature of such events, since the transverse momenta of the two LSPs are no longer oppositely aligned.

The dominant background from multi-jets is reduced by requiring a minimum azimuthal angle $\Delta \phi\left(\right.$ jet,$\left.E_{\mathrm{T}}^{\text {miss }}\right)$ between the jets and the $E_{\mathrm{T}}^{\text {miss }}$ in the event and by cutting on the ratio of $E_{\mathrm{T}}^{\text {miss }}$ and the effective mass. The remaining significant background contributions are measured in control regions (CR) and extrapolated to the SRs using transfer factors (TF). In the case of the irreducible $Z(\rightarrow v v)+$ jets background two CRs are used, one with an isolated photon selection, and one with a dilepton selection requiring the invariant mass of the two leptons to be inside a window around the $\mathrm{Z}$ boson mass. The photon $p_{\mathrm{T}}$ or $\mathrm{Z}$ boson $p_{\mathrm{T}}$ respectively are then added to the $E_{\mathrm{T}}^{\text {miss }}$ of the event to mimic the $E_{\mathrm{T}}^{\text {miss }}$ in the SRs. The TFs are derived from theoretically determined cross-section ratios of the processes $Z(\rightarrow v v)+$ jets and $\gamma+$ jets and from simulation. The multijet background is estimated from a CR with inverted $\Delta \phi$ selection. The corresponding TFs are determined with a data-driven technique based on the smearing of well-measured low $E_{\mathrm{T}}^{\text {miss }}$ events 
by the known detector resolution. The leptonic $t \bar{t}$, single-top and $\mathrm{W}$ backgrounds are estimated from CRs requiring leptons. In these events, the transverse mass between lepton and $E_{\mathrm{T}}^{\text {miss }}$ is required to be within $30-100 \mathrm{GeV}$, and a b-tag selection or veto is applied when isolating top or $\mathrm{W}$ processes respectively. The leptons are counted as jets in these CRs to mimic the $\mathrm{W}$ and top events in the SRs. The TFs are derived from simulation. All TFs and event yields in the CRs are input to a global likelihood fit which is performed separately for each SR.

The number of observed events in each SR is in good agreement with the SM expectation. No significant excess is observed. The $\mathrm{CL}_{S}$ method is used to derive $95 \%$ confidence level exclusion limits using for each set of parameters of a given model the SR with the best expected sensitivity. Fig. 1 (left) shows the exclusion limits in the $m_{\tilde{q}}-m_{\tilde{g}}$ plane for a set of simplified models in which the masses of the first and second generation squarks are assumed to be degenerate and the mass of the LSP is set to 0. All other SUSY particles including third generation squarks are decoupled by assigning masses of $5 \mathrm{TeV}$. Squark masses up to $\sim 1380 \mathrm{GeV}$ and gluino masses up to $\sim 840$ $\mathrm{GeV}$ are excluded. Fig. 1 (right) shows the $m_{0}-m_{1 / 2}$ plane of the CMSSM/MSUGRA slice detailed in the introduction, where gluinos and squarks of equal mass are excluded at $\sim 1400 \mathrm{GeV}$. The red lines in both figures indicate the observed limits, the dashed-blue lines the median expected limits, and the dotted blue lines the $\pm 1 \sigma$ variation on the expected limits.

\section{Searches in final states with 6-9 jets and missing transverse energy}

The search for SUSY in events with 6-9 jets and missing transverse energy [4] covers supersymmetric scenarios with high jet multiplicities, which may arise from quark emission in supersymmetric cascade decays, production of $\mathrm{W}$ or $\mathrm{Z}$ bosons decaying to hadrons, or from $\mathrm{QCD}$ radiation. Examples of particular phenomenological interest include models where squarks are significantly heavier than gluinos. In such models gluino pair production may dominate and subsequent decays to top quarks can produce large jet multiplicities. The events are selected with two multi-jet trig-
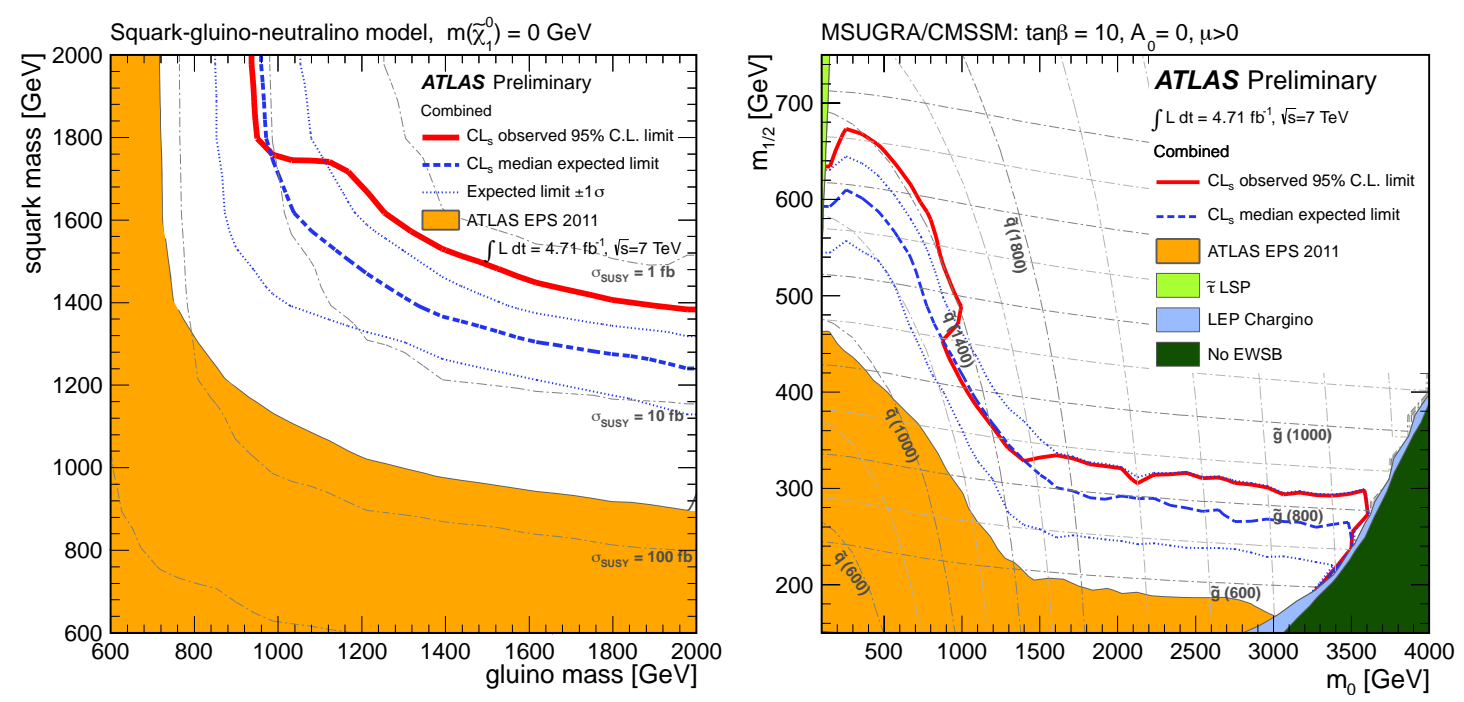

Figure 1: Exclusion limits of the 2-6 jets $+E_{T}^{\text {miss }}$ analysis for a simplified gluino-squark-neutralino model (left) and CMSSM/MSUGRA scenarios (right) as indicated in the text [3]. 
gers with different jet $p_{\mathrm{T}}$ thresholds and multiplicities. Based on these triggers three SRs requiring 6-8 jets with $p_{\mathrm{T}}>55 \mathrm{GeV}$, and three SRs requiring 7-9 jets with $p_{\mathrm{T}}>80 \mathrm{GeV}$ are defined. Events with electrons or muons are vetoed. The final SR selection is $E_{\mathrm{T}}^{\text {miss }} / \sqrt{H_{\mathrm{T}}}>4 \mathrm{GeV}^{1 / 2}$, where $H_{\mathrm{T}}$ is the scalar sum of the transverse momenta of jets with $p_{\mathrm{T}}>40 \mathrm{GeV}$.

The dominant background in this analysis is from multi-jets (including fully hadronic $t \bar{t}$ ) due to the absence of a requirement on the minimum azimuthal angle $\Delta \phi\left(\right.$ jet, $\left.E^{\text {miss }}\right)$ as well as softer $E_{\mathrm{T}}^{\text {miss }}$ selections than for the analysis described in section 2. The multi-jet background is estimated with templates of the $E_{\mathrm{T}}^{\text {miss }} / \sqrt{H_{\mathrm{T}}}$ distribution which are obtained at lower jet multiplicities. The underlying assumption that $E_{\mathrm{T}}^{\text {miss }} / \sqrt{H_{\mathrm{T}}}$ is independent of jet multiplicity is fulfilled within the uncertainties. The shape templates are then normalized to the SRs in sideband regions defined by $E_{\mathrm{T}}^{\text {miss }} / \sqrt{H_{\mathrm{T}}}<1.5 \mathrm{GeV}^{1 / 2}$ after subtraction of the leptonic backgrounds. The dominant leptonic backgrounds, i.e. semi- and fully-leptonic $t \bar{t}$ and $\mathrm{W} / \mathrm{Z}+$ jets, are measured in dedicated CRs. The $t \bar{t}$ and $\mathrm{W}+$ jets CRs are formed by selecting events with exactly one muon. The transverse mass between the muon and the missing transverse momentum is required to be within $30<m_{\mathrm{T}}<$ $100 \mathrm{GeV}$. In addition, a b-tag requirement or veto is imposed to distinguish between the $t \bar{t}$ and $\mathrm{W}+$ jets processes respectively. The muons are treated as jets and SR selections are applied to mimic the backgrounds in the SRs. The leptonic $Z+j e t s ~ C R$ is defined by requiring a muon pair with invariant mass in proximity of the $\mathrm{Z}$ boson mass. The calculated $\mathrm{Z}$ boson $p_{\mathrm{T}}$ is added to the $E_{\mathrm{T}}^{\mathrm{miss}}$ and again SR selections are applied. The CR event yields are extrapolated to the SRs with TFs determined from simulation.

No significant excess of events over the SM expectation is observed in any of the SRs. $95 \%$ confidence level exclusion limits are derived using for each set of parameters of a given model the SR with the best expected sensitivity. Fig. 2 (left) shows the exclusion contour for a simplified model with gluino pair production and subsequent decays via virtual stops to $t \bar{t}$ and neutralino. Gluino masses below $870 \mathrm{GeV}$ are excluded for neutralino masses up to $100 \mathrm{GeV}$ in this model. Fig. 2 (right) shows the plane of universal scalar and gaugino masses of the CMSSM/MSUGRA
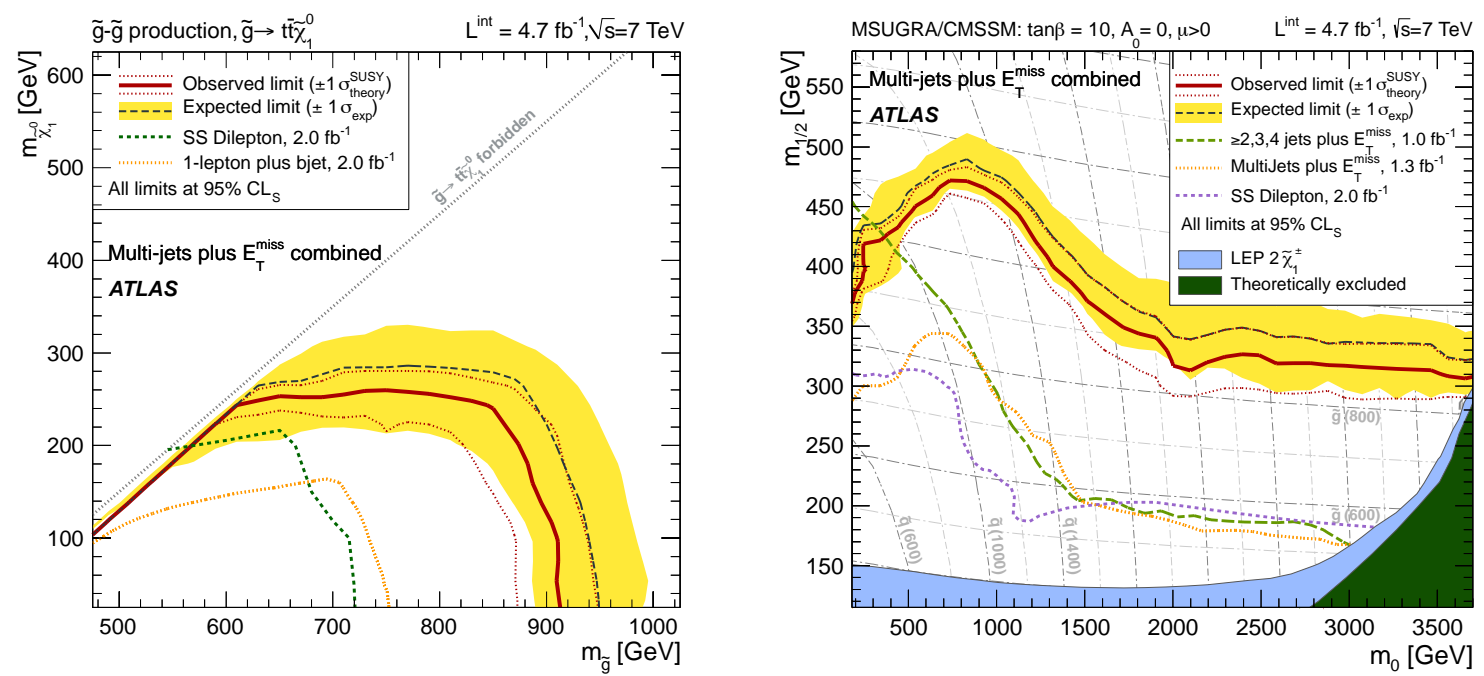

Figure 2: Exclusion limits of the 6-9 jets $+E_{T}^{\text {miss }}$ analysis for a simplified gluino-neutralino model (left) and CMSSM/MSUGRA scenarios (right) as indicated in the text [4]. 
slice described in the introduction. The analysis substantially extends the previous exclusion limits for $m_{0}>500 \mathrm{GeV}$. For larger values of $m_{0}$, the analysis becomes independent of the squark mass, and the lower bound on the gluino mass is extended to approximately $840 \mathrm{GeV}$ for large $m_{\tilde{q}}$. The $\pm 1 \sigma$ band surrounding the expected limit in both figures shows the variation anticipated from statistical fluctuations and systematic uncertainties on SM and signal processes. The lines on either side of the observed limit represent the $\pm 1 \sigma$ uncertainty on the expected signal cross-section.

\section{Searches in final states with 1 lepton, 2-4 jets, and missing transverse energy}

Supersymmetry searches with leptons in addition to jets and $E_{\mathrm{T}}^{\text {miss }}$ in the final state are complementary to the fully hadronic searches. While the acceptance in leptonic search channels is reduced due to SM branching ratios and the requirement of gauginos in the SUSY decay, the rejection of hadronic backgrounds is significantly enhanced due to the lepton selection. In this analysis [5] three mutually exclusive SRs are defined, two SRs with high- $p_{\mathrm{T}}$ lepton selections above $25 \mathrm{GeV}$ and $20 \mathrm{GeV}$ for electrons and muons respectively, and one complementary low- $p_{\mathrm{T}} \mathrm{SR}$ with lepton transverse momenta between 7-25 GeV and 6-20 GeV for electrons and muons respectively. In the two high- $p_{\mathrm{T}}$ SRs events with three and four jets are selected respectively, while the low- $p_{\mathrm{T}} \mathrm{SR}$ requires a jet multiplicity of two. The low- $p_{\mathrm{T}} \mathrm{SR}$ is designed for an improved sensitivity to SUSY models with compressed spectra, where all decay products are expected to be soft and initial state radiation may help to select the event. Further selection criteria are the transverse mass between the lepton and $E_{\mathrm{T}}^{\text {miss }}$ in the event, the effective mass defined as the scalar sum of the transverse momenta of the lepton and jets in the event, the ratio of $E_{\mathrm{T}}^{\text {miss }}$ and effective mass, and $E_{\mathrm{T}}^{\text {miss }}$ itself.

The dominant backgrounds, from $t \bar{t}$ and $\mathrm{W}+$ jets processes, are measured in enriched CRs. The multi-jet background is determined with an independent data-driven technique and the remaining minor backgrounds such as Z+jets, single-top, and di-boson production are taken from simulation. The estimates are input to a simultaneous likelihood fit which uses the shape of the jet multiplicity distributions in the $t \bar{t}$ and $\mathrm{W}+$ jets CRs. The estimated event yields in the CRs are extrapolated to the SRs with TFs taken from simulation. The fit is overconstrained such that some systematic uncertainties are reduced in the fit using data. The validity of the background estimates is tested in validation regions that lie between the CRs and the SRs.

As in the previously presented analyses no significant excess over the SM expectation has been observed in the any of the SRs. Exclusion limits are derived from an additional fit in the SRs. Fig. 3 (top) shows the exclusion limits for a simplified model with gluino pair-production followed by 1 -step decays of the type $\tilde{g} \rightarrow q \bar{q} / \tilde{\chi}_{1}^{ \pm} \rightarrow q \bar{q} / W^{ \pm} \tilde{\chi}_{1}^{0}$, where the mass of the chargino lies midway between that of the gluino and the neutralino. On the left the $95 \%$ exclusion limits are shown for the statistically combined high- $p_{\mathrm{T}} \mathrm{SRs}$ and on the right for the low- $p_{\mathrm{T}} \mathrm{SR}$. The colour code denotes the excluded cross-section. The high- $p_{\mathrm{T}}$ SRs (left) perform well in the bulk of the $m_{\tilde{g}}-m_{\tilde{\chi}_{1}^{0}}$ plane, whereas the low- $p_{\mathrm{T}} \mathrm{SR}$ (right) significantly improves the sensitivity in the compressed mass region close to the diagonal. The bottom part of Fig. 3 shows the exclusion contour for the same slice of CMSSM/MSUGRA parameter space as in the previous analyses. Squarks and gluinos of equal mass are excluded for $m<1200 \mathrm{GeV}$ which constitutes a significant improvement over previous analyses. The $\pm 1 \sigma$ lines surrounding the expected limit in both figures show the variation anticipated from statistical fluctuations and systematic uncertainties on SM and signal processes. 

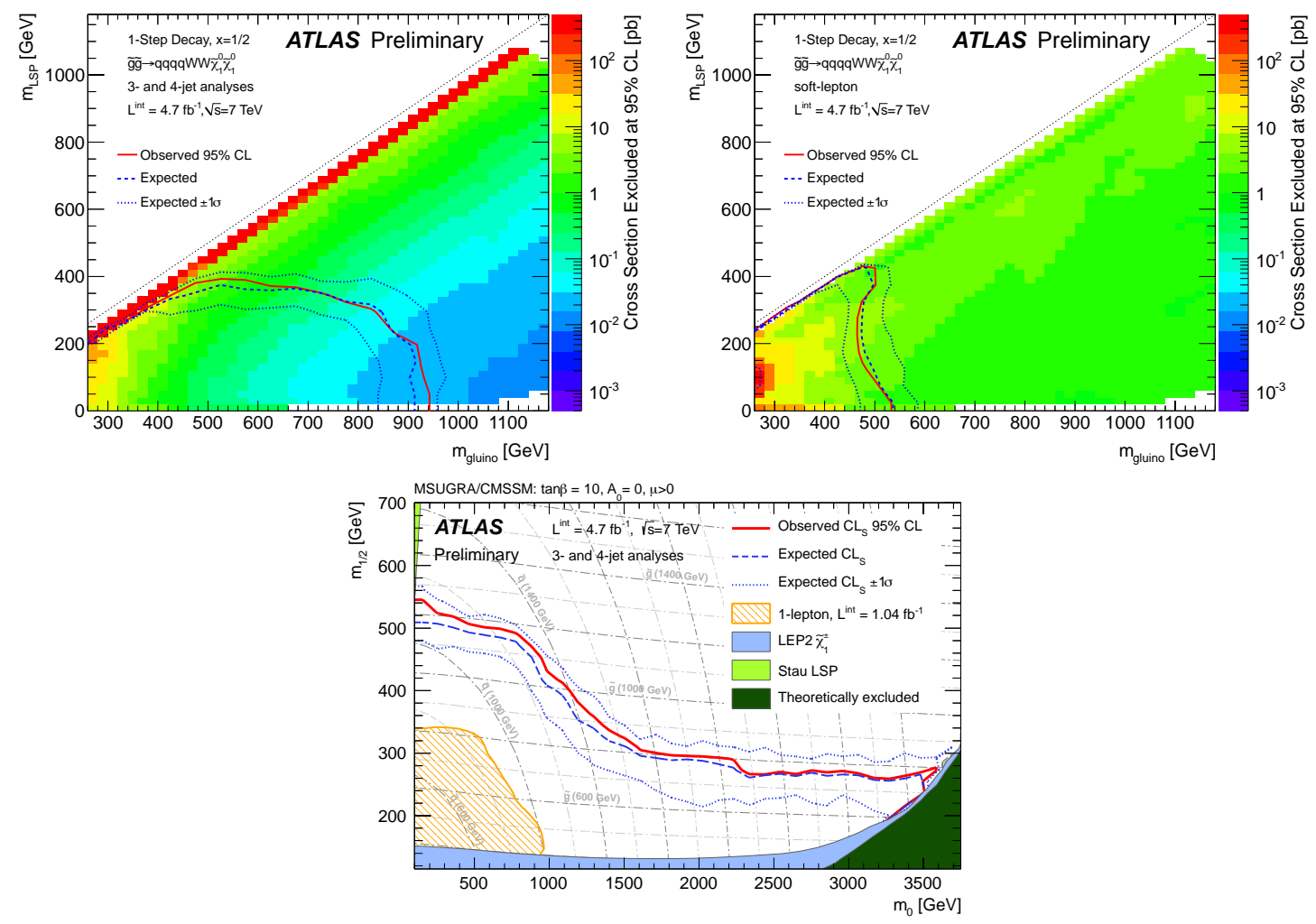

Figure 3: Exclusion limits of the 1-lepton $+2-4$ jets $+E_{T}^{\text {miss }}$ analysis for a simplified 1-step gluino-charginoneutralino model (top) and CMSSM/MSUGRA scenarios (bottom) as indicated in the text [5].

\section{Conclusion}

Three inclusive searches for gluinos and first and second generation squarks have been presented using the full 2011 ATLAS dataset of $4.7 \mathrm{fb}^{-1}$ at $7 \mathrm{TeV}$ proton-proton collision energy. No significant excess over the SM expectation is found in any of these analyses. Previous exclusion limits on CMSSM/MSUGRA and a variety of simplified models have been increased significantly in particular for compressed SUSY and multi-jet scenarios.

\section{References}

[1] ATLAS Collaboration JINST 3 (2008) S08003.

[2] A. H. Chamseddine, R. L. Arnowitt, and P. Nath Phys.Rev.Lett. 49 (1982) 970. R. Barbieri, S. Ferrara, and C. A. Savoy Phys.Lett. B119 (1982) 343. L. E. Ibanez Phys.Lett. B118 (1982) 73.

[3] ATLAS Collaboration ATLAS-CONF-2012-033.

https://cdsweb.cern.ch/record/1432199.

[4] ATLAS Collaboration JHEP 1207 (2012) 167, arXiv:1206.1760 [hep-ex] .

[5] ATLAS Collaboration ATLAS-COM-CONF-2012-038.

https: / / cdsweb. cern. ch/record/1426982. 